N. E. Piskunov, W. W. Weiss, D. F. Gray, eds.

\title{
Observational Evidence for the Stratification of Chemical Abundances in Stellar Atmospheres
}

\author{
T. Ryabchikova
}

Institute of Astronomy, Russian Academy of Sciences, Pyatnitskaya 48, 119017 Moscow, Russia

Institute for Astronomy, University of Vienna, Türkenschanzstrasse 17, 1180 Vienna, Austria

G.A. Wade

Department of Physics, Royal Military College of Canada, PO Box 17000, Station "Forces" Kingston, Ontario, K7K 7B4, Canada

F. LeBlanc

Département de physique et d'astronomie, Université de Moncton, Moncton, New Brunswick, E1A 3E9, Canada

\begin{abstract}
.
We present spectroscopic and photometric observational evidence for abundance stratification in stellar atmospheres. Attention is given to chemically peculiar (Ap) stars in which magnetic fields stabilize the atmosphere, allowing diffusion processes to establish abundance stratification during the early stages of star's life. The results of recent empirical modelling of chemically stratified atmospheres are briefly discussed, and a comparison is given with the predictions of self-consistent atmospheric models which include radiative diffusion.

The importance of abundance stratification analysis is demonstrated for rapidly oscillating (roAp) stars in which the amplitudes of the radial velocity pulsations are different for the lines of different elements in different ionization stages. We also demonstrate that chemical stratification has important effects on the Zeeman Stokes $I Q U V$ profiles of Ap stars, indicating that stratification must be taken into account in detailed modelling of their magnetic fields.
\end{abstract}

\section{Introduction}

The atmospheres of most stars are believed to be chemically homogeneous. In lower main sequence stars convective mixing homogenizes the atmospheres, while in the upper main sequence stars (which are usually rapid rotators) rotationinduced mixing or turbulent winds oppose separation processes. However, there exists a large group of stars whose atmospheres are sufficiently stable to allow chemical element separation. These stars comprise the different peculiar classes of the upper main sequence stars with radiative atmospheres. 
A variety of peculiarities are observed in the form of underabundances (He in He-weak stars; CNO in magnetic Ap stars; Ca \& Sc in Am stars, metals in $\lambda$ Boo stars) as well as overabundances (He in He-rich stars, Si in Si-stars; $\mathrm{Mn}, \mathrm{Hg}$ and other heavy elements in $\mathrm{HgMn}$ stars; iron peak ( $\mathrm{Cr}$ in particular), rare-earths elements in most of Ap and Am stars, see also Weiss et al. 2002). Peculiar stars are usually slow rotators or they possess a global magnetic field, which stabilizes their atmospheres and favours a mutual diffusion of all the elements due to different separation processes. Radiative diffusion, proposed by Michaud (1970) and developed in numerous subsequent papers, is the most sophisticated theory of element separation which has been able to explain the common chemical peculiarities among the different groups of peculiar stars. Babel (1992) performed diffusion calculations for $\mathrm{Ca}, \mathrm{Ti}, \mathrm{Cr}, \mathrm{Mn}$ and $\mathrm{Sr}$ in the atmosphere of a magnetic star with $T_{\text {eff }}=8500 \mathrm{~K}$ and with a weak stellar wind, and provided theoretical distributions of these elements versus optical depth in the stellar atmosphere. His simple step-like vertical abundance distributions (or abundance profiles) (Babel 1994) were used in empirical stratification studies of the peculiar stars $\beta$ CrB (Wade et al. 2001) and $\gamma$ Equ (Ryabchikova et al. 2002). Another class of stars where separation processes play an important role is the white dwarfs. Recently, self-consistent model atmospheres including the effects of diffusion on the atmospheric structure have been calculated for white dwarfs (Dreizler \& Wolff 1999) and blue horizontal-branch stars (Hui-Bon-Hoa, LeBlanc, \& Hauschildt 2000). These models predict abundance profiles in the atmospheres for a large number of chemical elements, along with corresponding changes in the atmospheric structure.

There are a number of observational features in spectroscopy and spectrophotometry of chemically peculiar and related stars which cannot be reproduced with the best available chemically homogeneous atmospheric models. In Section 2 we present spectroscopic evidence for abundance stratification and a comparison between the predictions of the best available self-consistent diffusion model atmospheres and the observations will be given in Section 3. Some important applications of the stratified atmospheres will be discussed in Section 4 .

\section{Spectroscopy}

Empirical modelling of abundance stratification is mainly based on the differential analysis of spectral lines (line profiles) formed at different optical depths. We list here the main observational facts which require the introduction of vertical abundance inhomogeneities in model stellar atmospheres.

- An impossibility to fit the wing and the core of strong spectral lines with the same abundance. This phenomenon is observed in upper MS peculiar stars through the entire temperature range of chemical peculiarity.

1. He I line profiles in hot peculiar stars $\left(T_{\text {eff }}=15000-22000 \mathrm{~K}\right)$ A nice illustration of this feature is given in Figs. 5,6 of the paper by Bonifacio, Castelli, \& Hack (1995). In the hot chemically peculiar field horizontal-branch B-type star Feige 86 the He abundance required to fit the He I line wings gives theoretical line cores which are too strong 

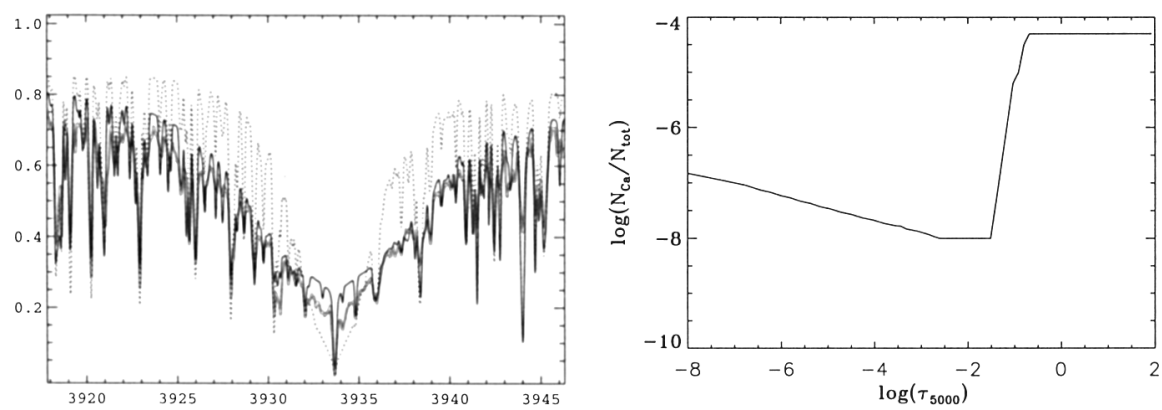

Figure 1. Ca II K line in the spectrum of $\gamma$ Equ (left panel). Observations are shown by a double line, the synthetic spectrum using the best homogeneous $\mathrm{Ca}$ abundance is shown by a dotted line, while the synthetic spectrum calculated with an empirically-derived $\mathrm{Ca}$ abundance profile (right panel) is shown by solid line.

compared to the observations, while a perfect fit is obtained in the spectrum of the normal B star $\iota$ Her using a chemically homogeneous atmospheric model of the same effective temperature. The observed anomaly in He I line profiles was used to derive an empirical vertical He distribution in the atmospheres of He-strong (Bohlender 1989) and He-weak (Farthmann et al. 1994) stars.

2. Ga III $\lambda 1495$ line profile in spectra of $3 \mathrm{HgMn}$ stars $\left(T_{\text {eff }}=12000-13000\right.$ K). Smith (1995 - Figure 1 of his paper) found that a satisfactory fit to the observed $\mathrm{Ga}$ II and $\mathrm{Ga}$ III UV resonance line profiles requires a $\mathrm{Ga}$ overabundance of up to 4 dex in atmospheric layers above $\log \tau_{5000}=0$ with a very steep abundance gradient.

3. Ca II K line in Ap stars with $T_{\text {eff }} \leq 9000 \mathrm{~K}$. Babel $(1992,1994)$ was the first to calculate $\mathrm{Ca}$ abundance stratification in Ap stars, and these calculations were applied to the magnetic Ap star 53 Cam and later to a sample of 28 Ap stars. Babel stressed that 'Ca II K and $\mathrm{H}$ lines in Ap stars give precious information about abundance stratification'. This is illustrated in Figure 1 for the Ap star $\gamma$ Equ taken from the paper by Ryabchikova et al. (2002). In all of the stars investigated by Babel calcium is underabundant in the upper atmospheric layers, with a steep abundance jump of up to 2 dex between $-1.0 \leq \log \tau_{5000} \leq-0.5$ towards deeper layers.

4. Ba II $\lambda 6141$ line in one of the coolest and craziest Ap stars - the famous Przybylski's star (HD 101065) - with $T_{\text {eff }}=6600 \mathrm{~K}$. An impossibility to fit this line with a single $\mathrm{Ba}$ abundance was mentioned by Cowley et al. (2000). Figure 2 and Figure 3 (right panels) show a comparison between the observed Ba II $\lambda 6141$ line profile and the synthetic spectra calculated with homogeneous and stratified $\mathrm{Ba}$ abundances.

- A violation of LTE ionization balance. In stratified atmospheres spectral lines of different ions are formed in different atmospheric layers 

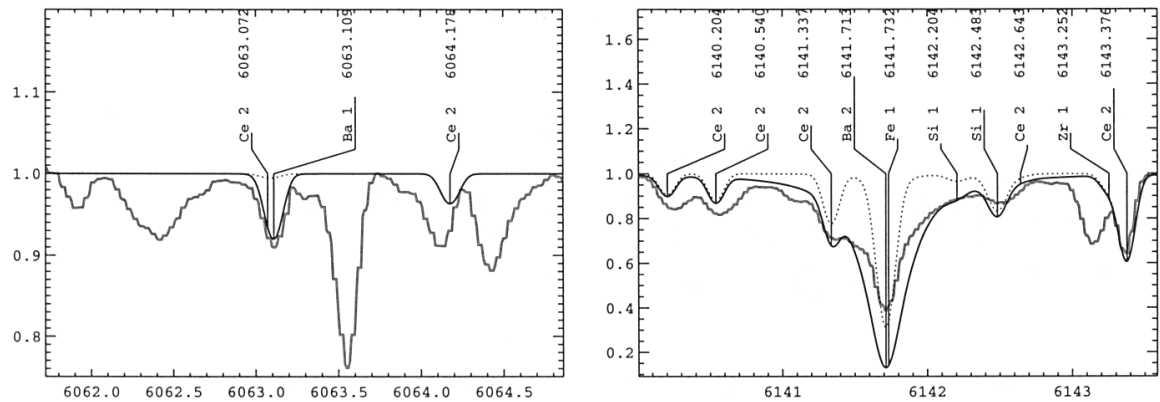

Figure 2. Ba I (left panel) and Ba II (right panel) lines in the spectrum of Przybylski's star = HD 101065 (double line). Dotted and $\mathrm{s}$ lines represent synthetic spectra calculated with homogeneous $\mathrm{Ba}$ abundances which fits the line core $\left(\log \left(B a / N_{t o t}\right)=-9.20\right)$ and the line wing $\left(\log \left(B a / N_{\text {tot }}\right)=-7.60\right)$ of the Ba II $\lambda 6141$ line.
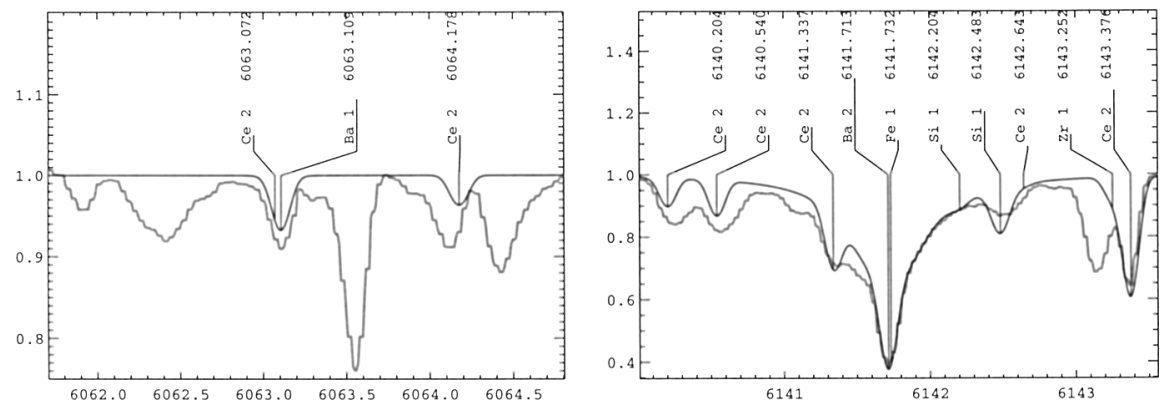

Figure 3. The same as in Figure 2 but for a stratified Ba abundance profile similar to that shown for Ca with $\log \left(B a / N_{\text {tot }}\right)=-10.0$ above $\log \tau_{5} 000=-1.0$ and with $\log \left(B a / N_{\text {tot }}\right)=-7.0$ below this optical depth.

(as compared to chemically homogeneous atmospheres) which results in an apparent ionization imbalance in classical LTE abundance studies: e.g. Zr II-Zr III, Pt I-Pt II-Pt III, Hg I-Hg II-Hg III in the HgMn star $\chi$ Lup (Lecrone et al. 1999); REE II-REE III (rare-earth elements) in roAp stars (Ryabchikova et al. 2001). Spectral lines of the higher ions usually give higher abundance. In roAp stars $\mathrm{Pr}$ and Nd were proposed to be concentrated in a thin uppermost atmospheric cloud. An opposite case is observed for e.g. Ba in Przybylski's star. (Figures 2, 3 with Ba I and Ba II lines). Here $\mathrm{Ba}$ is concentrated towards the deeper layers.

- A disagreement between the abundances derived from strong and weak lines of the same ion. Depending on the details of the stratification profile this effect may be similar or opposite to the effects of microturbulence. Concentration of an element in deep atmospheric layers weakens strong lines and enhances weak lines, an effect in contrast to that of microturbulence. 

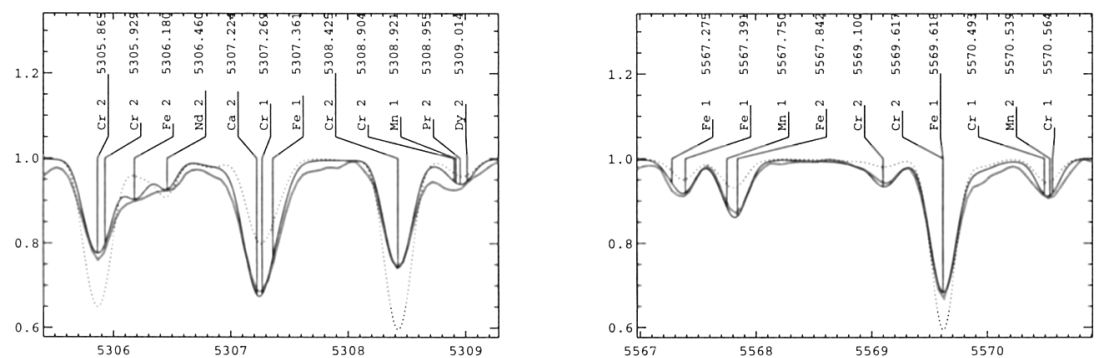

Figure 4. A comparison between the observed $\mathrm{Cr}$ II line profiles with low excitation $E_{\mathrm{i}}=4.1 \mathrm{eV}$ (left panel- double line) and high excitation $E_{\mathrm{i}}=10.9 \mathrm{eV}$ (right panel-double line) and synthetic spectrum calculations with the best homogeneous $\mathrm{Cr}$ abundance (dotted line) and $\mathrm{Cr}$ abundance stratification from Ryabchikova et al. (2002) (solid line).

- An unexpected behaviour of high-excitation $\left(E_{\mathrm{i}}>10 \mathrm{eV}\right)$ lines of the ionized iron peak elements.

1. In cool Ap stars $\mathrm{Cr}$ II and Fe II lines with $E_{\mathrm{i}}>10 \mathrm{eV}$ are unusually strong for the corresponding effective temperature (Figure 4). This fact was used in an empirical study of abundance stratification in the Ap stars $\beta$ CrB (Wade et al. 2001) and $\gamma$ Equ (Ryabchikova et al. 2002).

2. In a few HgMn and normal B-type stars high-excitation lines of Mn II, and possibly $\mathrm{Cr}$ II, $\mathrm{Ti}$ II were discovered in emission (Sigut, Landstreet, \& Shorlin 2000; Wahlgren \& Hubrig 2000). Although the main mechanism responsible for this emission is non-LTE excitation, it is not possible to explain the variety of the observed intensities in $\mathrm{HgMn}$ stars, from pure strong absorption $(\kappa \mathrm{Cnc})$ to strong emission (3 Cen A), without assuming Mn stratification in the atmosphere in a simple step function approximation (Sigut 2001a - Figure 1; Sigut 2001b).

- Different abundances obtained from the lines of the same ions formed at a priori different optical depths, for example, before and after Balmer jump, in UV and visual spectral regions, $\mathrm{Cr}$ II lines of Multiplet 30 in the wing of $\mathbf{H} \beta$ line. The last possibility was studied by Savanov, Kochukhov, \& Tsymbal (2001) in spectra of a set of $\mathrm{HgMn}, \mathrm{Ap}$ and Am stars. An empirical Cr stratification in the atmosphere of $\beta \mathrm{CrB}$ obtained by this method is in a good agreement with the $\mathrm{Cr}$ stratification derived by Wade et al. (2001, see also poster D7 of this conference). A comparative study of Mn II lines (Alecian 1982, Monier \& Alecian poster D25 of this conference) and Ga II lines (Lanz et al. 1993) in the UV and optical in HgMn stars led them to the tentative conclusion about possible stratification effects in their atmospheres, indicating that $\mathrm{Mn}$ has to be concentrated in the upper atmospheric layers (recall that this is also required to explain Mn II emission lines in HgMn stars). 
- Isotopic anomalies. It is a famous ${ }^{3} \mathrm{He} /{ }^{4} \mathrm{He}$ anomaly observed mainly in helium-peculiar stars and in the hotter HgMn stars (Hartoog \& Cowley 1979, Zakharova \& Ryabchikova 1996, Bohlender et al. poster D3 of this conference), as well as isotopic anomalies of heavy elements, for example, Hg (Woolf \& Lambert 1999; Hubrig, Castelli, \& Mathys 1999; Proffitt et al. 1999), and Pt (Kalus et al. 1998; Bohlender, Dworetsky, \& Jomaron 1998; Hubrig et al. 1999) in HgMn stars. The effects of gravitational settling, mass loss, radiatively-driven diffusion and light-induced drift (Atutov \& Shalagin 1988; LeBlanc \& Michaud 1993; Aret \& Sapar 2002) contribute to the formation of these anomalies, although no single mechanism could be able to explain all elemental and isotopic anomalies observed.

From the analysis of the empirical/(partially theoretical) abundance stratification studies considered above we may conclude:

- The separation processes responsible for abundance stratification in nonmagnetic peculiar stars ( $\mathrm{HgMn}, \mathrm{Am})$ appear to create enhancement of the abundances of most elements except He in the outer atmospheric layers.

- On the other hand, in magnetic stars with $T_{\text {eff }}<10000 \mathrm{~K}$ element accumulation occurs below $\log \tau_{5000}=-1$. The only exception may be rare-earth elements in roAp stars.

- The vertical chemical abundance distributions in stellar atmosphere can be reasonably approximated using a single-step model.

\section{Comparison with the self-consistent diffusion model predictions}

Most of previous diffusion calculations were oriented to one single chemical element and were based on LTE model atmospheres without taking into account the feedback of the strong abundance anomalies on the atmospheric structure. Self-consistent diffusion models which are presently under development give us stratification profiles for many chemical elements (from $\mathrm{H}$ to La in the models of Ap stars). These models allow prediction of the observed abundance anomalies, spectral line profiles and energy distributions for different groups of stars from white dwarfs to cool Ap stars.

Dreizler \& Wolff (1999, also see Dreizler in these proceedings) showed that a self-consistent diffusion model calculated for the DA white dwarf G 191-B2B reproduces the complete EUVE spectrum without any additional photospheric or interstellar absorbers or mass loss (see Figures 6 and 7 of Dreizler \& Wolff 1999). This model can reproduce the UV lines of Fe, and to a lesser extent, those of Ni. Larger disagreements remain for $\mathrm{C}, \mathrm{N}, \mathrm{O}$, and $\mathrm{Si}$.

Recently, Hui-Bon-Hoa et al. (2000) have shown that self-consistent diffusion models provide much better agreement with many observational features for blue horizontal-branch stars: jumps in the observed $(u-(u-y))$ colour diagrams, gaps in $(\mathrm{V}-(\mathrm{U}-\mathrm{V}))$ diagrams, and higher spectroscopic gravities as compared with the evolutionary ones. These atmospheric models are calculated with a modified version of the PHOENIX (Hauschildt et al. 1999) code. Models are now being developed for other types of stars such as Ap (see Figure 5) and HgMn stars. 


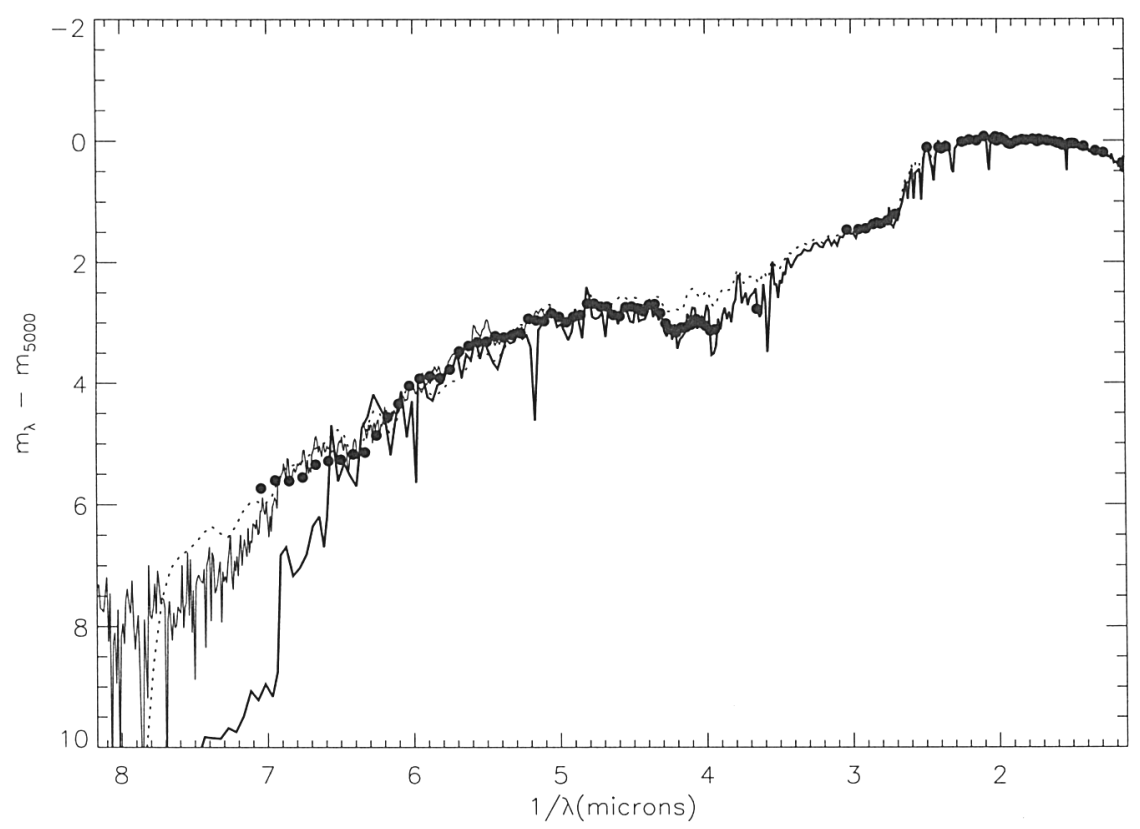

Figure 5. $\quad \beta \mathrm{CrB}$ : optical spectrophotometry by Pyper \& Adelman (1985), UV spectrophotometry S2/68 TD-1 (Jamar et al. 1976) - filled circles, and low resolution IUE SWP44998 spectrum - thin line, are compared with the model flux calculations: the best homogeneous Atlas9 8000g43_ODF model (thick line), stratified Phoenix 7700g40 model (dotted line).

Hack et al. (1997) explained an observed UV flux excess in the Ap star $\beta$ $\mathrm{CrB}$ by invoking the presence of a $\lambda$ Boo-type companion of the same effective temperature. However, no trace of the purported secondary's lines were found in high-resolution spectra. The first self-consistent diffusion model for an Ap star $(\beta \mathrm{CrB})$ is presented in these proceedings (Wade et al. poster D7). This model naturally explains the observed UV flux excess (Figure 5) and fits reasonably well the observed line profiles for many elements (Figure 6). However, it fails to fit the $\mathrm{H} \alpha$ line profile. The theoretical vertical distributions of $\mathrm{Cr}$ and $\mathrm{Fe}$ in the atmosphere of $\beta \mathrm{CrB}$ are characterized by abundance jumps within the line forming region similar to those obtained empirically. However, the vertical position of the abundance jump is shifted to deeper atmospheric layers in the theoretical model. Cowley et al. (2001) found that various cool Ap stars exhibited anomalous $\mathrm{H} \alpha$ line cores.(Figure 7). Kochukhov et al. (poster D17 of this conference) studied this anomaly on a larger set of stars and could reproduce it empirically by introducing a temperature jump between $-1.0<\log \tau_{5000}<-$ 3.0. Model atmosphere diffusion calculations predict temperature jumps at the atmospheric layers where most elements accumulate (see poster D7 of this conference), but somewhat deeper than the empirical jump proposed by Kochukhov 

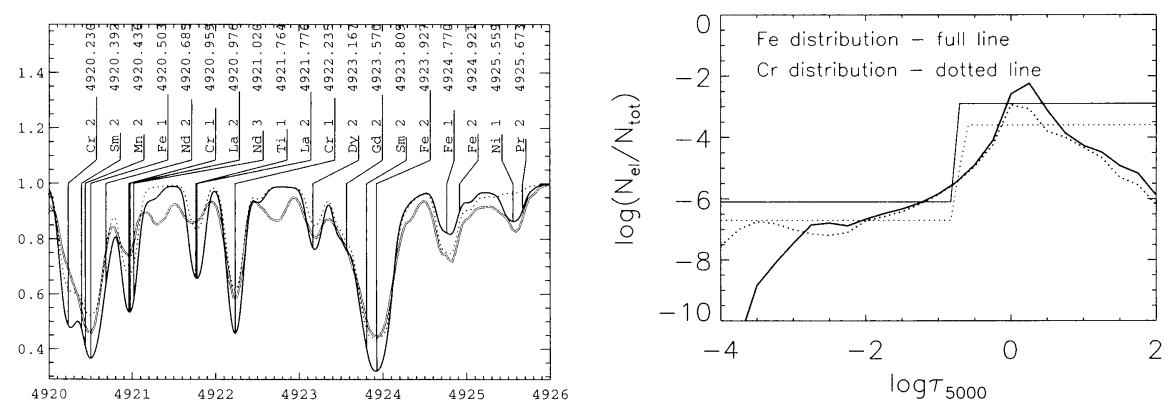

Figure 6. A part of the spectrum of $\beta \mathrm{CrB}$ (left panel). Observations are shown by a double line, synthetic spectra calculated with the empirical and theoretical abundance distributions are shown by dotted and solid lines, respectively. A comparison of the empirically-derived $\mathrm{Cr}$ and Fe distributions (thin lines) and the corresponding distributions obtained with the self-consistent diffusion model (thick lines) are given in the right panel.

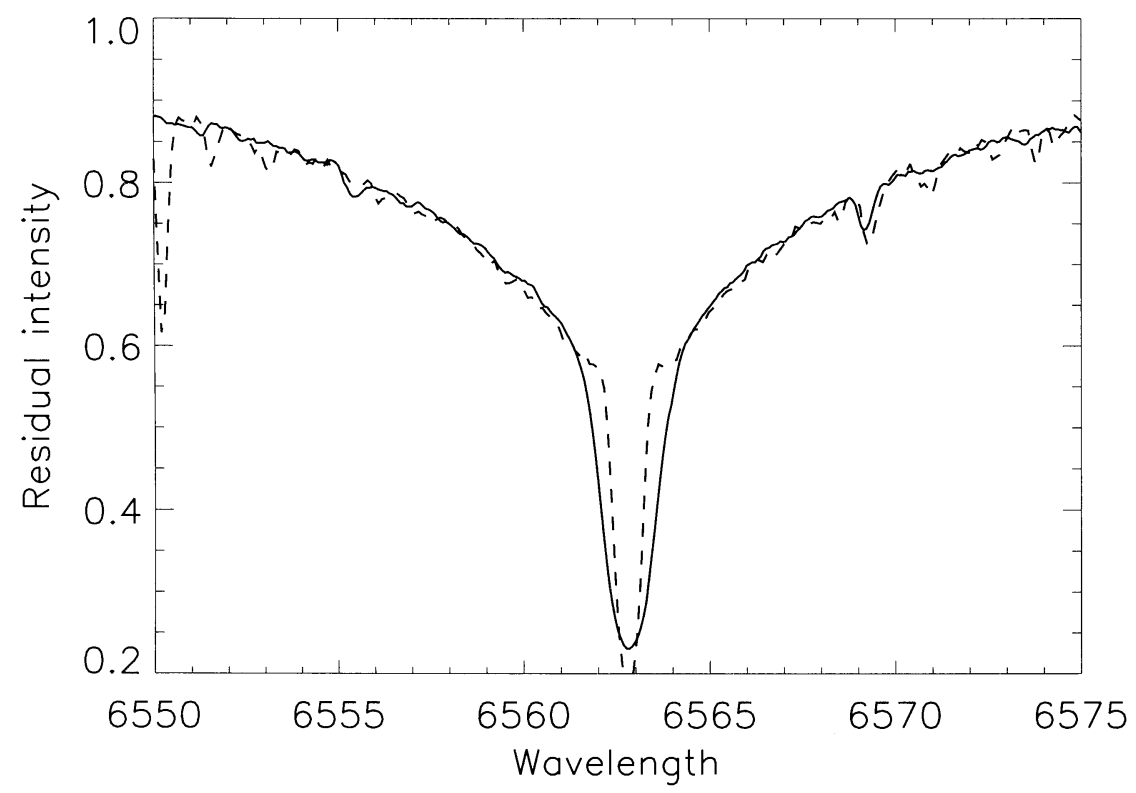

Figure 7. "Core-wing anomaly" in cool Ap stars. A comparison of the $\mathrm{H} \alpha$ line in the spectrum of the normal late-type A star HD 32115 (solid line) with the $\mathrm{H} \alpha$ line in the spectrum of roAp star HD 24712 (dashed line). These stars have the same atmospheric parameters $\left(T_{\text {eff }}, \log g\right)$ according to classical analyses. 

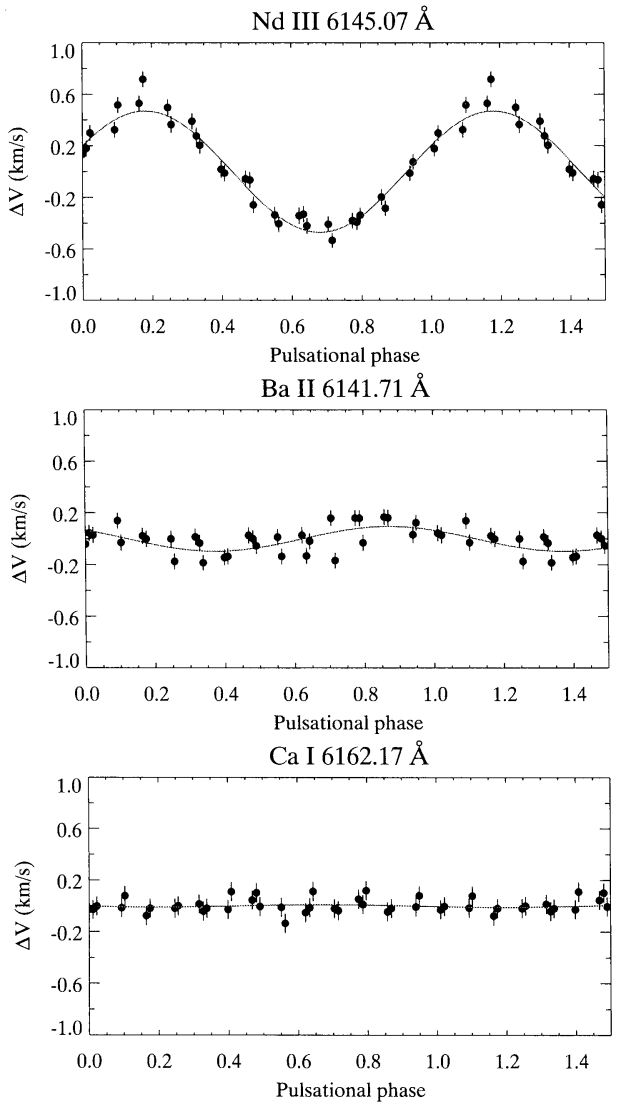

Figure 8. Pulsational radial velocity variations from three spectral lines of the same intensity in $\gamma$ Equ.

et al. We suspect that the "core-wing anomaly" observed in cool Ap stars is caused by stratification effects, and that improved self-consistent diffusion model atmospheres will explain it.

Current self-consistent diffusion models for magnetic Ap stars do not include magnetic field effects. Alecian \& Stift (2002) have shown that desaturation of spectral lines due to magnetic splitting changes significantly the radiative accelerations. The magnetic field configuration itself is important as well.

\section{Importance of chemical stratification}

\subsection{Rapidly oscillating Ap stars and asteroseismology}

In a study of the pulsational phenomenon in cool Ap stars, Kochuchov \& Ryabchikova (2001a,b) found that lines of similar intensity in spectra of three roAp stars ( $\gamma$ Equ, $\alpha$ Cir and HD 83368) have radial velocity amplitudes varying from 0 to more than $1 \mathrm{~km} \mathrm{~s}^{-1}$. Figure 8 shows pulsational RV curves derived 

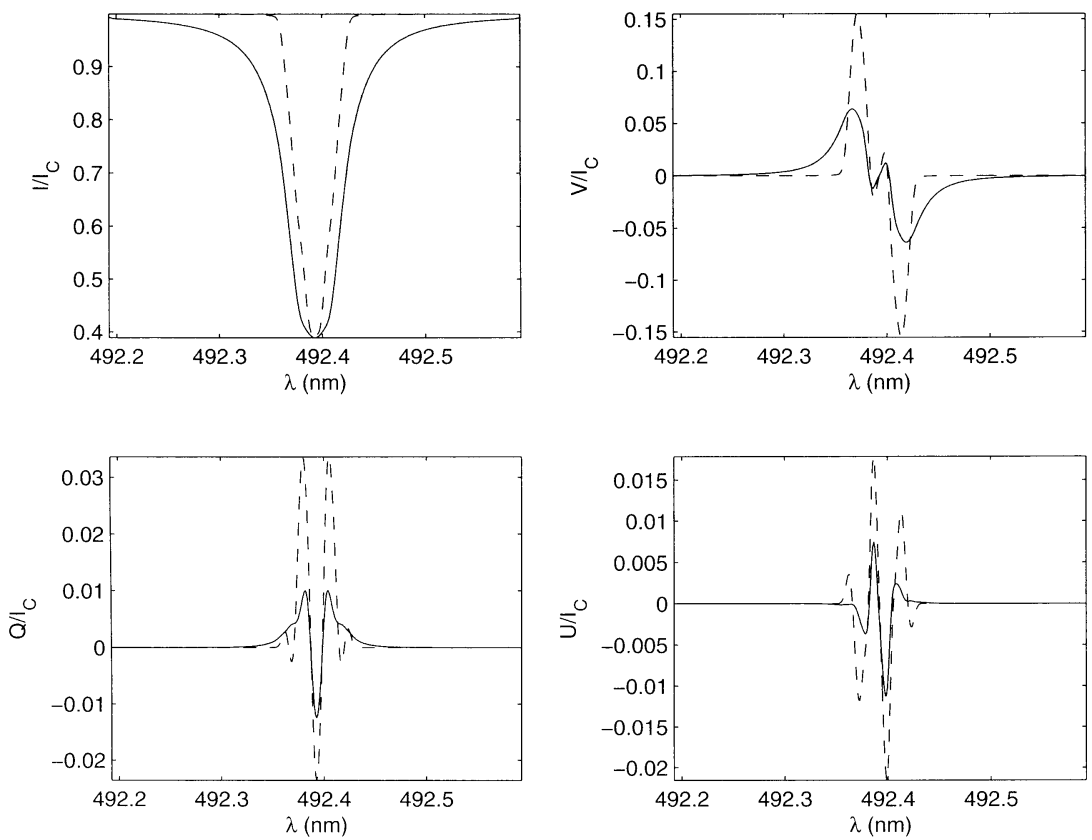

Figure 9. Calculations comparing Stokes $I Q U V$ profiles of Fe II $492.39 \mathrm{~nm}$ for uniform (dashed line) and stratified (solid line, using a single-step model similar to that of $\beta \mathrm{CrB}$ ) abundances, with $v \sin i=0 \mathrm{~km} \mathrm{~s}^{-1}$ and a dipolar magnetic field with $B_{\mathrm{d}}=10 \mathrm{kG}$. Note the very large differences in all four Stokes profiles, indicating that stratification must be taken into account when interpreting such observations. From Wade (2002, in prepration).

from three strong lines in the spectrum of $\gamma$ Equ. In chemically homogeneous atmospheres these lines are formed at the same optical depths. However, in the frame of the empirical stratification model suggested for $\gamma$ Equ, the pulsation amplitudes increase towards the upper atmospheric layers (Ryabchikova et al. 2002). Careful modelling of abundance stratification in roAp stars including non-LTE effects will provide extremely important information for pulsation theory of these stars.

\subsection{Stokes $I Q U V$ profiles and detailed magnetic fields modelling}

The modified shapes of line profiles shows that stratification modifies the line opacity distribution. Not only will these modifications lead to changes in the (Stokes $I$ ) line profile, it will also impact the shapes and intensities of the associated Stokes $V Q U$ profiles of magnetic stars. In Figure 9 we show how stratification can have very large and systematic effects on Stokes profiles. This may lead to inaccurate reconstructions of surface magnetic field configurations (using methods like MDI, Kochukhov \& Piskunov 2002) if these effects are not taken into account. 
Acknowledgments. TR acknowledges IAU, Fonds zur Förderung der wissenschaftlichen Forschung P14984 and Österreichische Nationalbank (Jubiläumsfonds $N r .7650$ ) for a financial support.

\section{Discussion}

COHEN: I call to your attention the $\mathrm{PhD}$ thesis of my former graduate student Brad Behr, where evidence for gravitational settling of radiative levitation is given for several elements in hot horizontal branch stars in globular clusters. The data spans a range of $T_{\text {eff }}$ and rotational velocities and represent a good set for theoretical modelling.

\section{References}

Alecian, G. 1982, A\&A, 107, 61

Alecian, G., \& Stift, M. J. 2002,A\&A, 387, 271

Aret, A., \& Sapar, A. 2002, AN, 323, 21

Atutov, S. N., \& Shalagin, A. M. 1988, Sov. Astron. Let, 14, 284

Babel, J. 1992, A\&A, 258, 449

Babel, J. 1994, A\&A, 283, 189

Bohlender, D.A. 1989, ApJ, 346, 459

Bohlender, D.A., Dworetsky, M. M., \& Jomaron, C. M. 1998, ApJ, 504, 533

Bonifacio, P., Castelli, F., \& Hack M. 1995, A\&AS, 110, 441

Cowley, C.R., Ryabchikova, T.A., Kupka, F., Bord, D.J., Mathys, G., \& Bidelman, W.P. 2000, MNRAS, 317, 299

Cowley, C. R., Hubrig, S., Ryabchikova, T.A., Mathys, G., Piskunov, N., \& Mittermayer, P. 2001, A\&A, 367, 939

Dreizler, S., \& Wolff, B. 1999, A\&A, 348, 189

Farthmann, M., Dreizler, S., Heber, U., \& Hunger, K. 1994, A\&A, 291, 919

Hack, M., Polosukhina, N. S., Malanushenko, V. P., \& Castelli, F. 1997, A\&A, 319,637

Hartoog, M. R., \& Cowley, A. P. 1979, ApJ, 228, 229

Hauschildt, P.H., Allard, F., \& Baron, E., 1999, ApJ, 512, 377

Hubrig, S., Castelli, F., \& Mathys, G. 1999, A\&A, 341, 190

Hui-Bon-Hoa, A., LeBlanc, F., \& Hauschildt, P.H. 2000, ApJ, 535, L43

Jamar, C., Macau-Hercot, D., Monfils, A., Thompson, G. I., Houziaux, L., \& Wilson, R. 1976, Ultraviolet Bright-Star Spectrophotometric Catalogue, ESA SR-27

Kalus, G., Johansson, S., Wahlgren, G. M., Leckrone, D.S., Thorne, A. P., \& Brandt, J. C. 1998, ApJ, 494, 792

Kochukhov, O., \& Ryabchikova, T. 2001a, A\&A, 374, 615

Kochukhov, O., \& Ryabchikova, T. 2001b, A\&A, 377, L22

Kochukhov, O., \& Piskunov, N. 2002, A\&A, 388, 868 
Lanz, T., Artru, M.-C., Didelon, P., \& Mathys, G. 1993, A\&A, 272, 465

LeBlanc, F., \& Michaud, G. 1993, ApJ, 408, 251

Leckrone, D. S., Proffitt, C. R., Wahlgren, G. M., Johansson, S. G., \& Brage, T. 1999, AJ, 117, 1454

Michaud, G. 1970, ApJ, 160, 641

Proffitt, C. R., Brage, T., Leckrone, D. S., Wahlgren, G. M., Brandt, J. C., \& Sansonetti, C. J. 1999, ApJ, 512, 942

Pyper, D. M., \& Adelman, S. J. 1985, A\&AS, 59, 369

Ryabchikova, T. A., Savanov, I.S., Malanushenko, V. P., \& Kudryavtsev, D. O. 2001, ARep., 45, 382

Ryabchikova, T., Piskunov, N., Kochukhov, O., Tsymbal, V., Mittermayer, P., \& Weiss, W. W. 2002, A\&A, 384, 545

Savanov, I. S., Kochukhov, O. P., \& Tsymbal, V. V. 2001, Ap, 44, 206

Sigut, T. A. A. 2001a, ApJ, 546, L115

Sigut, T. A. A. 2001b, A\&A, 377, L27

Sigut, T. A. A., Landstreet, J. D., \& Shorlin, S. L. S. 2000, ApJ, 530, L89

Smith, K.C. 1995, A\&A, 297, 237

Wade, G. A., Ryabchikova, T. A., Bagnulo, S., \& Piskunov, N. 2001, in Magnetic Fields Across the Hertzsprung-Russell Diagram, ed. G. Mathys, S. K. Solanki, \& D. T. Wickramasinghe, ASP Conf. Ser., 248, 341

Wahlgren, G. M., \& Hubrig, S. 2000, A\&A, 362, L13

Weiss, W.W., Rybachikova, T.A., Savanov, I., Piskunov, N., Tsymbal, V., Mittermayer, P., Martinez, P., Kochukhov, O., \& Nesvacil, N. 2002, ASP Conf. Ser., 259, 280

Woolf, V. M., \& Lambert, D. L. 1999, ApJ, 521, 414

Zakharova, L. A., \& Ryabchikova, T. A. 1996, 22, 152 